\title{
Quantum Cohomology of Grassmannians
}

\author{
ANDERS SKOVSTED BUCH ${ }^{\star}$ \\ Matematish Institut, Aarhus Universitet, Ny Munkegade, 8000 Århus C, Denmark. \\ e-mail:abuch@imf.au.dk
}

(Received: 18 October 2001; accepted: 14 March 2002)

\begin{abstract}
We give elementary proofs of the main theorems about the (small) quantum cohomology of Grassmannians, including the quantum Giambelli and quantum Pieri formulas, the rim-hook algorithm, the presentation, and a recent theorem of Fulton and Woodward about the minimal $q$-power which appears in a product of two Schubert classes.
\end{abstract}

Mathematics Subject Classifications (2000). 14N35 (primary); 14M15, 05E05 (secondary)

Key words. quantum cohomology, Gromov-Witten invariants, Grassmann varieties.

\section{Introduction}

The purpose of this paper is to give simple proofs of the main theorems about the (small) quantum cohomology ring of a Grassmann variety. This first of all includes Bertram's quantum versions of the Pieri and Giambelli formulas [1]. Bertram's proofs of these theorems required the use of quot schemes. Our proof of the quantum Pieri formula uses no moduli spaces and only the definition of Gromov-Witten invariants. In fact we show that this formula is a consequence of the classical Pieri formula. We then show that the quantum Giambelli formula follows immediately from the quantum Pieri formula together with the classical Giambelli formula and associativity of quantum cohomology $[13,17]$.

We also give a short proof of the Grassmannian case of a formula of Fulton and Woodward for the minimal $q$-power which appears in a quantum product of two Schubert classes [10]. In addition we supply a proof of a simple version of the rimhook algorithm [2] based on 'mod $n$ ' arithmetic which is due to F. Sottile [19]. Finally we recover the presentation of the quantum cohomology of Grassmannians [18, 21].

In this paper we only assume associativity of quantum cohomology and standard facts about the usual cohomology. The basic idea is that if a rational curve of degree $d$ passes through a Schubert variety in the Grassmannian $\operatorname{Gr}\left(l, \mathbb{C}^{n}\right)$ of $l$ dimensional subspaces of $\mathbb{C}^{n}$, then the linear span of the points of this curve gives rise to a point in $\operatorname{Gr}\left(l+d, \mathrm{C}^{n}\right)$ which must lie in a related Schubert variety. Remarkably, this simple idea can in many cases be used to conclude that no curves pass through three Schubert varieties in general position because the intersection of the

\footnotetext{
${ }^{\star}$ The author was partially supported by NSF Grant DMS-0070479.
} 
related Schubert varieties in $\operatorname{Gr}\left(l+d, \mathrm{C}^{n}\right)$ is empty. In particular, the quantum Giambelli formula can be deduced by knowing that certain Gromov-Witten invariants are zero, and in each case this follows because the codimensions of the related Schubert varieties add up to more than the dimension of $\operatorname{Gr}\left(l+d, \mathrm{C}^{n}\right)$. The same idea can be used to prove the quantum Monk's formula for flag varieties $S L_{n} / B[4,7]$ and even the quantum Pieri formula for partial flag varieties $S L_{n} / P[5$, 6]. In a paper [3] with A. Kresch and $H$. Tamvakis this idea will furthermore be applied to obtain a similar treatment of the quantum cohomology of Lagrangian and orthogonal Grassmannians $[14,15]$.

\section{Preliminaries}

Set $E=\mathbb{C}^{n}, X=\operatorname{Gr}(l, E)$, and $k=n-l$. Given a flag of subspaces $F_{1} \subset F_{2} \subset$ $\cdots \subset F_{n}=E$ and a partition $\lambda=\left(\lambda_{1} \geqslant \lambda_{2} \geqslant \cdots \geqslant \lambda_{l} \geqslant 0\right)$ with $\lambda_{1} \leqslant k$, we define the Schubert variety

$$
\Omega_{\lambda}\left(F_{\bullet}\right)=\left\{V \in X \mid \operatorname{dim}\left(V \cap F_{k+i-\lambda_{i}}\right) \geqslant i \forall 1 \leqslant i \leqslant l\right\} .
$$

The codimension of this variety is equal to the weight $|\lambda|=\sum \lambda_{i}$ of $\lambda$. We let $\Omega_{\lambda}$ denote the class of $\Omega_{\lambda}\left(F_{\bullet}\right)$ in the cohomology ring $H^{*} X=H^{*}(X ; Z)$. The Schubert classes $\Omega_{\lambda}$ form a basis for this ring. The partitions indexing this basis are exactly those where the Young diagram fits in an $l \times k$ rectangle.

The cohomology ring has the presentation $H^{*} X=\mathbb{Z}\left[\Omega_{1}, \ldots, \Omega_{k}\right] /\left(Y_{l+1}, \ldots, Y_{n}\right)$ where $Y_{p}=\operatorname{det}\left(\Omega_{1+j-i}\right)_{1 \leqslant i, j \leqslant p}$. Here we set $\Omega_{i}=0$ for $i<0$ or $i>k$ for convenience. In this presentation the class $\Omega_{\lambda}$ is given by the Giambelli formula

$$
\Omega_{\lambda}=\operatorname{det}\left(\Omega_{\lambda_{i}+j-i}\right)_{1 \leqslant i, j \leqslant l} .
$$

This is usually deduced (cf. [8]) from the Pieri formula, which states that

$$
\Omega_{i} \cdot \Omega_{\lambda}=\sum \Omega_{v}
$$

where the sum is over all partitions $v$ which can be obtained by adding $i$ boxes to the Young diagram of $\lambda$ with no two in the same column.

Recall that the degree of a rational curve $f: \mathbb{P}^{1} \rightarrow \mathbb{P}^{N}$ is equal to the number of points in the inverse image by $f$ of a general hyperplane in $\mathbb{P}^{N}$. The degree of a map $f: \mathrm{P}^{1} \rightarrow X$ is the degree of the composition of $f$ with the Plücker embedding $X \subset \mathbb{P}\left(\bigwedge^{l} E\right)$ which maps a point $V \in X$ to $v_{1} \wedge \cdots \wedge v_{l}$ for any basis $\left\{v_{1}, \ldots, v_{l}\right\}$ of $V$. Now let $\lambda, \mu$, and $v$ be three partitions contained in an $l \times k$ rectangle, and let $d \geqslant 0$ be an integer such that $|\lambda|+|\mu|+|v|=l k+\mathrm{d} n$. The Gromov-Witten invariant $\left\langle\Omega_{\lambda}, \Omega_{\mu}, \Omega_{\nu}\right\rangle_{d}$ is defined as the number of rational curves of degree $d$ on $X$, which meet all of the Schubert varieties $\Omega_{\lambda}\left(F_{\bullet}\right), \Omega_{\mu}\left(G_{\bullet}\right)$, and $\Omega_{\nu}\left(H_{\bullet}\right)$ for general flags $F_{\bullet}$, $G_{\bullet}, H_{\bullet}$, up to automorphisms of $\mathbb{P}^{1}$. A simple proof that this number is well defined is given in [1]. If $|\lambda|+|\mu|+|v| \neq l k+\mathrm{d} n$, then $\left\langle\Omega_{\lambda}, \Omega_{\mu}, \Omega_{\nu}\right\rangle_{d}=0$.

The (small) quantum cohomology ring $Q H^{*} X=Q H^{*}(X ; Z)$ of $X$ is a Z $Z[q]$-algebra which is isomorphic to $H^{*} X \otimes_{\mathrm{Z}} \mathrm{Z}[q]$ as a module over Z $[q]$. In this ring we have Schubert classes $\sigma_{\lambda}=\Omega_{\lambda} \otimes 1$. The ring structure on $Q H^{*} X$ is defined by 


$$
\sigma_{\lambda} \cdot \sigma_{\mu}=\sum_{v, d \geqslant 0}\left\langle\Omega_{\lambda}, \Omega_{\mu}, \Omega_{v^{v}}\right\rangle_{d} q^{d} \sigma_{v}
$$

where $v^{\vee}=\left(k-v_{l}, k-v_{l-1}, \ldots, k-v_{1}\right)$ is the partition for the dual Schubert class of $\Omega_{v}$. It is a nontrivial fact that this defines an associative ring structure [13, 17] (see also [9]). Notice that the definition implies that $Q H^{*} X$ is a graded ring where $\sigma_{\lambda}$ has degree $|\lambda|$ and $q$ has degree $n$. Furthermore, the map $Q H^{*} X /(q) \rightarrow H^{*} X$ which sends $\sigma_{\lambda}$ to $\Omega_{\lambda}$ is an isomorphism of rings, so the quantum ring is a deformation of the usual cohomology ring.

Siebert and Tian have given a presentation of this ring which is similar to the above presentation of the cohomology ring [18]. We will comment on this presentation towards the end of this paper. For now we will start from the basic assumption that the quantum ring is a well defined associative ring, and aim towards proving generalizations of the Pieri and Giambelli formulas stated above to reveal its structure.

\section{The Span and Kernel of a Curve}

Our main new tool is the following definition. If $Y$ is any subvariety of $X=\operatorname{Gr}(l, E)$ we define the span of $Y$ to be the smallest subspace of $E$ containing all the $l$-dimensional spaces given by points of $Y$. Similarly we define the kernel of $Y$ to be the largest subspace of $E$ contained in all the spaces given by points of $Y$.

LEMMA 1. Let $C$ be a rational curve of degree $d$ in $X$. Then the span of $C$ has dimension at most $l+d$ and the kernel of $C$ has dimension at least $l-d$.

Proof. Let $C$ be the image of a regular function $f: \mathbb{P}^{1} \rightarrow X$ of degree $d$, and let $S \subset E \otimes \mathcal{O}_{X}$ be the tautological subbundle on $X$. Then $f^{*} S=\bigoplus_{i=1}^{l} \mathcal{O}_{\mathrm{P}^{\mathrm{p}}}\left(-a_{i}\right)$ for integers $a_{i} \geqslant 0$ with sum $d$, and $f$ is given by an inclusion $\bigoplus_{i=1}^{l} \mathcal{O}_{\mathbb{P}^{1}}\left(-a_{i}\right) \subset E \otimes \mathcal{O}_{\mathbb{P}^{1}}$, i.e. a point $p \in \mathbb{P}^{1}$ is mapped to the fiber over $p$ of the image of this bundle map. If $(s: t)$ are homogeneous coordinates on $\mathrm{P}^{1}$ then $\Gamma\left(\mathcal{O}_{\mathrm{p}^{1}}\left(a_{i}\right)\right)$ has the basis $\left\{s^{j} t^{a_{i}-j}\right\}_{0 \leqslant j \leqslant a_{i}}$, so each map $\mathcal{O}_{\mathrm{p}^{1}}\left(-a_{i}\right) \rightarrow E \otimes \mathcal{O}_{\mathbb{P}^{1}}$ has the form

$$
\sum_{j=0}^{a_{i}} \alpha_{j} s^{-j} t^{j-a_{i}} \mapsto \sum_{j=0}^{a_{i}} v_{j}^{(i)} \otimes \alpha_{j}
$$

for vectors $v_{j}^{(i)} \in E$ (which will depend on the chosen identification of $f^{*} S$ with $\left.\bigoplus_{i=1}^{l} \mathcal{O}_{\mathrm{P}^{1}}\left(-a_{i}\right)\right)$. The span of $C$ must therefore be contained in the span of the set $\left\{v_{j}^{(i)}\right\}$ which has cardinality $\sum\left(1+a_{i}\right)=l+d$. On the other hand, at least $l-d$ of the integers $a_{i}$ must be zero, and the kernel of $C$ contains the span of the corresponding vectors $v_{0}^{(i)}$.

The above lemma can also be obtained by proving that any regular function $f: \mathbb{P}^{1} \rightarrow X$ can be written as $f(t)=f_{1}(t) \wedge \cdots \wedge f_{l}(t)$ for maps $f_{i}: \mathbb{P}^{1} \rightarrow \mathbb{P}(E)$. This is what we did until A. Kresch showed us the simpler argument given here. 
If $\lambda$ is a partition and $d$ a nonnegative integer, we let $\hat{\lambda}$ denote the partition obtained by removing the leftmost $d$ columns from the Young diagram of $\lambda$, i.e. $\hat{\lambda}_{i}=\max \left(\lambda_{i}-d, 0\right)$.

LEMMA 2. Let $C \subset X$ be a rational curve of degree $d \leqslant k$ and let $W \subset E$ be an $l+d$ dimensional subspace containing the span of $C$. If $\lambda$ is a partition such that $C \cap \Omega_{\lambda}\left(F_{\bullet}\right) \neq \emptyset$ then $W$ belongs to the Schubert variety $\Omega_{\hat{\lambda}}\left(F_{\bullet}\right)$ in $\operatorname{Gr}(l+d, E)$.

Proof. Let $V \in C \cap \Omega_{\lambda}\left(F_{\bullet}\right)$. Since $V \subset W$, the Schubert conditions on $V$ imply that $\operatorname{dim}\left(W \cap F_{k+i-\lambda_{i}}\right) \geqslant i$ for all $i$, which says exactly that $W$ belongs to $\Omega_{\hat{\lambda}}\left(F_{\bullet}\right)$.

Similarly, if $K \subset E$ is an $l-d$ dimensional subspace contained in the kernel of $C$, then $K \in \Omega_{\bar{\lambda}}\left(F_{\bullet}\right) \subset \operatorname{Gr}(l-d, E)$, where $\bar{\lambda}=\left(\lambda_{d+1}, \ldots, \lambda_{l}\right)$ is the result of removing the top $d$ rows of $\lambda$. Therefore, the condition that a curve meets a given set of Schubert varieties in $X$ implies that intersections of related Schubert varieties in $\operatorname{Gr}(l+d, E)$ and in $\operatorname{Gr}(l-d, E)$ are not empty, which is a statement about the usual cohomology of these spaces. As we shall see, this simple idea is sufficient to compute GromovWitten invariants in many important cases.

\section{The Quantum Pieri Formula}

We start with the following quantum version of the Pieri formula [1].

THEOREM 1 (Bertram). If $\lambda$ is contained in an $l \times k$ rectangle and $p \leqslant k$ then

$$
\sigma_{p} \cdot \sigma_{\lambda}=\sum \sigma_{\mu}+q \sum \sigma_{v}
$$

where the first sum is over all partitions $\mu$ such that $|\mu|=|\lambda|+p$ and $k \geqslant \mu_{1} \geqslant$ $\lambda_{1} \geqslant \mu_{2} \geqslant \lambda_{2} \geqslant \cdots \geqslant \mu_{l} \geqslant \lambda_{l}$, and the second sum is over all partitions $v$ such that $|v|=|\lambda|+p-n$ and $\lambda_{1}-1 \geqslant v_{1} \geqslant \lambda_{2}-1 \geqslant v_{2} \geqslant \cdots \geqslant \lambda_{l}-1 \geqslant v_{l} \geqslant 0$.

Recall that the length $\ell(\lambda)$ of a partition $\lambda$ is the number of non-zero parts of $\lambda$. Notice that the second sum in the theorem is nonzero only if $\ell(\lambda)=l$.

Proof. The first sum is dictated by the classical Pieri formula. Notice that this classical case is equivalent to the following statement. If $\alpha$ and $\beta$ are partitions such that $|\alpha|+|\beta|+p=l k$ then

$$
\left\langle\Omega_{\alpha}, \Omega_{\beta}, \Omega_{p}\right\rangle_{0}= \begin{cases}1 & \text { if } \alpha_{i}+\beta_{j} \geqslant k \text { for } i+j=l \text { and } \alpha_{i}+\beta_{j} \leqslant k \text { for } i+j=l+1 ; \\ 0 & \text { otherwise. }\end{cases}
$$

Now suppose $|\alpha|+|\beta|+p=l k+d n$ for some $d \geqslant 1$ and let $C$ be a rational curve of degree $d$ in $X$ which meets each of the varieties $\Omega_{\alpha}\left(F_{\bullet}\right), \Omega_{\beta}\left(G_{\bullet}\right)$, and $\Omega_{p}\left(H_{\bullet}\right)$ for general flags $F_{\bullet}, G_{\bullet}, H_{\bullet}$. Notice that $d n \leqslant l k+k$ which implies that $d \leqslant k$. Let $W \subset E$ be a subspace of dimension $l+d$ which contains the span of $C$. Then $W \in \operatorname{Gr}(l+d, E)$ lies in the intersection $\Omega_{\hat{\alpha}}\left(F_{\bullet}\right) \cap \Omega_{\hat{\beta}}\left(G_{\bullet}\right) \cap \Omega_{\hat{p}}\left(H_{\bullet}\right)$ where $\hat{\alpha}$ and $\hat{\beta}$ are the results of removing the leftmost $d$ columns from $\alpha$ and $\beta$, and 
$\hat{p}=\max (p-d, 0)$. Since the flags $F_{\bullet}, G_{\bullet}, H_{\bullet}$ are general, this implies that $|\hat{\alpha}|+|\hat{\beta}|+\hat{p} \leqslant(l+d)(k-d)$. Since we also have

$$
|\hat{\alpha}|+|\hat{\beta}|+\hat{p} \geqslant|\alpha|+|\beta|-2 l d+p-d=(l+d)(k-d)+d^{2}-d
$$

we deduce that $d=1$ and $\ell(\alpha)=\ell(\beta)=l$. Using this, the quantum Pieri formula becomes equivalent to the statement that if $|\alpha|+|\beta|+p=l k+n$ then $\left\langle\Omega_{\alpha}, \Omega_{\beta}, \Omega_{p}\right\rangle_{1}=1$ if $\alpha_{i}+\beta_{j} \geqslant k+1$ for $i+j=l+1$ and $\alpha_{i}+\beta_{j} \leqslant k+1$ for $i+j=l+2$; otherwise $\left\langle\Omega_{\alpha}, \Omega_{\beta}, \Omega_{p}\right\rangle_{1}=0$. In other words, the quantum Pieri formula states that $\left\langle\Omega_{\alpha}, \Omega_{\beta}, \Omega_{p}\right\rangle_{1}=\left\langle\Omega_{\hat{\alpha}}, \Omega_{\hat{\beta}}, \Omega_{\hat{p}}\right\rangle_{0}$ where the right hand side is a coefficient of the classical Pieri formula for $\operatorname{Gr}(l+1, E)$.

If $\left\langle\Omega_{\hat{\alpha}}, \Omega_{\hat{\beta}}, \Omega_{\hat{p}}\right\rangle_{0}$ is zero then the space $W$ can't exist, so neither can $C$. On the other hand, if $\left\langle\Omega_{\hat{\alpha}}, \Omega_{\hat{\beta}}, \Omega_{\hat{p}}\right\rangle_{0}=1$ then there exists a unique subspace $W \subset E$ of dimension $l+1$ which is contained in the intersection $\Omega_{\hat{\alpha}}\left(F_{\bullet}\right) \cap \Omega_{\hat{\beta}}\left(G_{\bullet}\right) \cap \Omega_{\hat{p}}\left(H_{\bullet}\right)$. Furthermore, since the flags are general, $W$ must lie in the interior of each of these Schubert varieties. In particular, each of the spaces $V_{1}=W \cap F_{n-\alpha_{l}}$ and $V_{2}=W \cap G_{n-\beta_{l}}$ have dimension $l$. Notice also that $V_{1} \in \Omega_{\alpha}\left(F_{\bullet}\right)$ and $V_{2} \in \Omega_{\beta}\left(G_{\bullet}\right)$. Since $\Omega_{\alpha}\left(F_{\bullet}\right) \cap$ $\Omega_{\beta}\left(G_{\bullet}\right)=\emptyset$ we deduce that $V_{1} \neq V_{2}$, so $K=V_{1} \cap V_{2}$ has dimension $l-1$. We conclude that the only rational curve of degree 1 in $X$ which meets the Schubert varieties for $\alpha, \beta$, and $p$ is the line $\mathrm{P}(W / K)$ of $l$-dimensional subspaces between $K$ and $W$.

\section{The Quantum Giambelli Formula}

Now set $\sigma_{i}=0$ for $i<0$ and for $k<i<n$. The quantum Giambelli formula states that the classical formula (2) continues to be valid if all Schubert classes are replaced with the corresponding quantum Schubert classes [1].

THEOREM 2 (Bertram). If $\lambda$ is a partition contained in an $l \times k$ rectangle, then the Schubert class $\sigma_{\lambda}$ in $Q H^{*} \operatorname{Gr}(l, E)$ is given by $\sigma_{\lambda}=\operatorname{det}\left(\sigma_{\lambda_{i}+j-i}\right)_{1 \leqslant i, j \leqslant l}$.

Proof. We claim that if $0 \leqslant i_{j} \leqslant k$ for $1 \leqslant j \leqslant l$ then $\sigma_{i_{1}} \cdot \sigma_{i_{2}} \cdots \sigma_{i_{l}}=$ $\left(\Omega_{i_{1}} \cdot \Omega_{i_{2}} \ldots \Omega_{i_{l}}\right) \otimes 1$, i.e. no $q$-terms show up when the first product is expanded in the quantum ring. Using induction, this can be established by proving that if $\ell(\mu)<l$ then the expansion of $\sigma_{i} \cdot \sigma_{\mu}$ involves no $q$-terms and no partitions of lengths greater than $\ell(\mu)+1$. The claim therefore follows from Theorem 1. Since the determinant of the quantum Giambelli formula is a signed sum of products of the form $\sigma_{i_{1}} \cdot \sigma_{i_{2}} \cdots \sigma_{i_{l}}$, we conclude from the classical Giambelli formula (2) that $\operatorname{det}\left(\sigma_{\lambda_{i}+j-i}\right)=$ $\operatorname{det}\left(\Omega_{\lambda_{i}+j-i}\right) \otimes 1=\Omega_{\lambda} \otimes 1=\sigma_{\lambda}$ as required.

Notice that this proof uses only that no $q$-terms are contained in a product $\sigma_{i} \cdot \sigma_{\mu}$ when $\ell(\mu)<l$ (in addition to the classical Giambelli and Pieri formulas). In fact, Theorem 1 could be replaced with the following lemma.

LEMMA 3. Let $\lambda$ and $\mu$ be partitions contained in an $l \times k$ rectangle such that $\ell(\lambda)+\ell(\mu) \leqslant l$. Then $\sigma_{\lambda} \cdot \sigma_{\mu}=\left(\Omega_{\lambda} \cdot \Omega_{\mu}\right) \otimes 1$. 
Proof. If $d \geqslant 1$ and $v$ is a partition such that $|\lambda|+|\mu|+|v|=l k+n d$, then any intersection $\Omega_{\hat{\lambda}}\left(F_{\bullet}\right) \cap \Omega_{\hat{\mu}}\left(G_{\bullet}\right) \cap \Omega_{\hat{\nu}}\left(H_{\bullet}\right)$ of general Schubert varieties in $\operatorname{Gr}(l+d, E)$ must be empty since $|\hat{\lambda}|+|\hat{\mu}|+|\hat{v}| \geqslant|\lambda|+|\mu|+|v|-2 d l=l k+d k-d l>(l+d)$ $(k-d)$. This shows that $\left\langle\Omega_{\lambda}, \Omega_{\mu}, \Omega_{v}\right\rangle_{d}=0$.

A dual version of this lemma states that if $\lambda_{1}+\mu_{1} \leqslant k$, then $\sigma_{\lambda} \cdot \sigma_{\mu}=\left(\Omega_{\lambda} \cdot \Omega_{\mu}\right) \otimes 1$. This follows by replacing the span of a curve with its kernel in the above proof, or by using the duality isomorphism $Q H^{*} \operatorname{Gr}(l, E) \cong Q H^{*} \operatorname{Gr}\left(k, E^{*}\right)$.

\section{The Minimal Power of $q$ in a Quantum Product}

As a further demonstration of the use of the span of a rational curve in $X$, we will give a short proof of a recent theorem of Fulton and Woodward [10] in the case of Grassmannians. It generalizes the fact that any product $\sigma_{\lambda} \cdot \sigma_{\mu}$ in $Q H^{*} X$ is nonzero.

Given a partition $\lambda$ and an integer $d \geqslant 0$, we let $\hat{\bar{\lambda}}$ denote the partition obtained by removing the top $d$ rows and the leftmost $d$ columns from $\lambda$. Recall that a product $\Omega_{\lambda} \cdot \Omega_{\mu}$ is non-zero in $H^{*} \operatorname{Gr}(l, E)$ if and only if $\lambda_{i}+\mu_{j} \leqslant k$ for $i+j=l+1$.

THEOREM 3 (Fulton and Woodward). The smallest power of $q$ which appears in a product $\sigma_{\lambda} \cdot \sigma_{\mu}$ in $Q H^{*} X$ is equal to the smallest d for which $\Omega_{\hat{\lambda}} \cdot \Omega_{\mu} \neq 0$ in $H^{*} X$.

Proof. If $\sigma_{\lambda} \cdot \sigma_{\mu}$ contains $q^{d}$ times a Schubert class then some curve of degree $d$ meets each of the Schubert varieties $\Omega_{\lambda}\left(F_{\bullet}\right)$ and $\Omega_{\mu}\left(G_{\bullet}\right)$ where $F_{\bullet}$ and $G_{\bullet}$ are general flags. If $W \subset E$ has dimension $l+d$ and contains the span of this curve then $W$ lies in the intersection $\Omega_{\hat{\lambda}}\left(F_{\bullet}\right) \cap \Omega_{\hat{\mu}}\left(G_{\bullet}\right)$ in $\operatorname{Gr}(l+d, E)$. In particular this intersection is not empty which implies that $\Omega_{\hat{\lambda}} \cdot \Omega_{\hat{\mu}} \neq 0$ in $H^{*} \operatorname{Gr}(l+d, E)$. Since this is equivalent to $\Omega_{\hat{2}} \cdot \Omega_{\mu} \neq 0$ in $H^{*} X$, this proves the inequality ' $\geqslant$ ' of the theorem. (If $d>k$ then it is also true that $\Omega_{\hat{2}} \cdot \Omega_{\mu} \neq 0$.)

Now let $d$ be the smallest number for which $\Omega_{\hat{2}} \cdot \Omega_{\mu} \neq 0$. Notice that this implies that $\lambda$ contains a $d \times d$ rectangle, i.e. $\lambda_{d} \geqslant d$. Set $\alpha=\left(k+d-\lambda_{d}, \ldots, k+d-\lambda_{1}\right)$ and let $\beta$ be the partition given by $\beta_{i}=\max \left(d-\lambda_{l+1-i}, 0\right)$ for $1 \leqslant i \leqslant l$. If the Young diagram for $\lambda$ is put in the upper-left corner of an $l$ by $k+d$ rectangle, then $\alpha$ is the complement of $\lambda$ in the top $d$ rows of this rectangle, turned 180 degrees, and $\beta$ is the complement of $\lambda$ in the leftmost $d$ columns, also turned.

It follows from the Littlewood-Richardson rule that the product $\sigma_{\lambda} \cdot \sigma_{\beta}$ contains the class $\sigma_{\left(d^{\prime}\right)+\hat{\lambda}}=\sigma_{\left(d^{\prime}\right)} \cdot \sigma_{\hat{\lambda}}$ and that $\sigma_{\hat{\lambda}} \cdot \sigma_{\alpha}$ contains $\sigma_{\left(k^{d}\right), \hat{\lambda}}=\sigma_{\left(k^{d}\right)} \cdot \sigma_{\hat{\lambda}}$. Since the structure constants of $Q H^{*} X$ are all nonnegative, this implies that $\sigma_{\lambda} \cdot \sigma_{\beta} \cdot \sigma_{\alpha}$ contains the product $\sigma_{\left(d^{l}\right)} \cdot \sigma_{\left(k^{d}\right)} \cdot \sigma_{\hat{\lambda}}=q^{d} \sigma_{\hat{\lambda}}$. Since $\Omega_{\hat{\lambda}} \cdot \Omega_{\mu} \neq 0$ by assumption, we conclude that $\sigma_{\lambda} \cdot \sigma_{\mu} \cdot \sigma_{\alpha} \cdot \sigma_{\beta}$ contains $q^{d}$ times some Schubert class. In particular, at least one term of the product $\sigma_{\lambda} \cdot \sigma_{\mu}$ must involve a power of $q$ which is less than or equal to $d$. This proves the other inequality ' $\leqslant$ ' in the theorem. Notice that the identities we have used in this argument follow easily from Theorem 1 and the dual version of Lemma 3, combined with the classical Pieri rule. 


\section{The Rim-Hook Algorithm}

We will next recall how to carry out computations in the quantum cohomology ring of $X$. Let $c_{i} \in Q H^{*} X$ denote the $i$ th Chern class of the dual of the tautological subbundle $S \subset E \otimes \mathcal{O}_{X}$. This means that $c_{i}=\sigma_{\left(1^{i}\right)}$ for $0 \leqslant i \leqslant l$ and $c_{i}=0$ for $i<0$ and $i>l$. For $p \geqslant 1$ we then define $\sigma_{p}=\operatorname{det}\left(c_{1+j-i}\right)_{1 \leqslant i, j \leqslant p}$ in $Q H^{*} X$. Notice that for $p<n$ we have $\sigma_{p}=\Omega_{p} \otimes 1$, so this definition is compatible with our previous definition of $\sigma_{p}$. The definition implies that for every $m \geqslant 1$ we have

$$
\sum_{i=0}^{l}(-1)^{i} \sigma_{m-i} \sigma_{\left(1^{i}\right)}=0
$$

LEMMA 4. For any $p \geqslant k+1$ we have $\sigma_{p}=(-1)^{l-1} q \sigma_{p-n}$.

Proof. Both sides of the identity are zero for $k+1 \leqslant p<n$. It follows from (5) with $m=n$ that $\sigma_{n}+(-1)^{l} \sigma_{k} \sigma_{\left(1^{\prime}\right)}=0$, so $\sigma_{n}=(-1)^{l-1} \sigma_{k} \sigma_{\left(1^{\prime}\right)}=(-1)^{l-1} q$ by Theorem 1. For $p>n$ we finally obtain

$$
\sigma_{p}=\sum_{i=1}^{l}(-1)^{i-1} \sigma_{p-i} \sigma_{\left(1^{i}\right)}=(-1)^{l-1} q \sum_{i=1}^{l}(-1)^{i-1} \sigma_{p-n-i} \sigma_{\left(1^{i}\right)}=(-1)^{l-1} q \sigma_{p-n}
$$

by induction, which proves the lemma.

If $I=\left(I_{1}, I_{2}, \ldots, I_{p}\right)$ is any sequence of integers we set $\sigma_{I}=\operatorname{det}\left(\sigma_{I_{i}+j-i}\right)_{1 \leqslant i, j \leqslant p}$ in $Q H^{*} X$. This is compatible with our notation $\sigma_{\lambda}$ for partitions $\lambda$ by Theorem 2 . Any element $\sigma_{I}$ can be rewritten as zero or plus or minus $\sigma_{\mu}$ for some partition $\mu$ by moves of the form $\sigma_{I, a, a+1, J}=0$ and $\sigma_{I, a, b, J}=-\sigma_{I, b-1, a+1, J}$, which correspond to interchanging rows in the determinant defining $\sigma_{I}$. Notice that if $\lambda$ is a partition then we have the formal identity $\sigma_{\lambda}=\operatorname{det}\left(c_{\lambda_{i}^{\prime}+j-i}\right)$ where $\lambda^{\prime}$ is the conjugate partition of $\lambda$, obtained by mirroring the Young diagram for $\lambda$ in its diagonal. In particular, if $\ell(\lambda)>l$ then the top row of the latter determinant is zero, and $\sigma_{\lambda}=0$.

COROLLARY 1 . Let $\lambda$ be a partition with at most $l$ parts. For each $1 \leqslant j \leqslant l$, choose $I_{j} \in Z$ such that $I_{j} \equiv \lambda_{j}(\bmod n)$ and $j-l \leqslant I_{j}<j-l+n$. Then we have

$$
\sigma_{\lambda}=(-1)^{d(l-1)} q^{d} \sigma_{I} \in Q H^{*} X
$$

where $I=\left(I_{1}, \ldots, I_{l}\right)$ and $n d=|\lambda|-\sum I_{j}$.

Notice that the moves described above will rewrite the chosen element $\sigma_{I}$ to zero or plus or minus a basis element $\sigma_{\mu}$ of $Q H^{*} X$ with $\mu$ contained in an $l \times k$ rectangle. The corollary is equivalent to the dual rim-hook algorithm of [2]. The formulation in terms of reduction modulo $n$ is due to F. Sottile [19], who in turn was inspired by the work of Ravi, Rosenthal, and Wang [16]. This formulation gives an efficient algorithm to determine if two elements $\sigma_{\lambda}$ and $\sigma_{\mu}$ are identical. In particular it shows that $\sigma_{\lambda}=0$ if and only if $\lambda_{i}-i \equiv \lambda_{j}-j(\bmod n)$ for some $i \neq j$. 
Corollary 1 makes it easy to do computations in $Q H^{*} X$ (cf. [2, 11, 20] and [12, Ex. 13.35]). In order to expand a product $\sigma_{\lambda} \cdot \sigma_{\mu}$, one uses the classical LittlewoodRichardson rule to write $\sigma_{\lambda} \cdot \sigma_{\mu}=\sum c_{\lambda \mu}^{v} \sigma_{v}$ where the sum is over partitions $v$ of length at most $l$ and $c_{\lambda \mu}^{v}$ is the Littlewood-Richardson coefficient. Each of the elements $\sigma_{v}$ can then be reduced to a basis element of $Q H^{*} X$ by the corollary.

\section{Generators and Relations}

Finally, we show how to deduce Siebert and Tian's presentation of the quantum cohomology ring of $X$ [18] (see also [21]). We claim that

$$
Q H^{*} X=Z\left[c_{1}, \ldots, c_{l}, q\right] /\left(\sigma_{k+1}, \ldots, \sigma_{n-1}, \sigma_{n}+(-1)^{l} q\right) .
$$

In fact, all of the given relations vanish in $Q H^{*} X$ by Lemma 4. On the other hand, the proof of Lemma 4 together with Corollary 1 shows that the linear map $H^{*} X \otimes \mathbb{Z}[q] \rightarrow \mathbb{Z}\left[c_{1}, \ldots, c_{l}, q\right] /\left(\sigma_{k+1}, \ldots, \sigma_{n-1}, \sigma_{n}+(-1)^{l} q\right)$ which sends $\Omega_{\lambda} \otimes q^{d}$ to $q^{d} \sigma_{\lambda}$ is surjective, which proves the isomorphism. Siebert and Tian gave this presentation in its dual form $Q H^{*} X=Z\left[\sigma_{1}, \ldots, \sigma_{k}, q\right] /\left(\tilde{Y}_{l+1}, \ldots, \tilde{Y}_{n-1}, \tilde{Y}_{n}+(-1)^{k} q\right)$ where the $\tilde{Y}_{i}$ are defined inductively by $\tilde{Y}_{0}=1, \quad \tilde{Y}_{i}=0$ for $i<0$, and $\tilde{Y}_{i}=$ $\sum_{j=1}^{k}(-1)^{j-1} \sigma_{j} \tilde{Y}_{i-j}$ for $i>0$.

The presentation (6) can also be stated as $Q H^{*} X=\mathbb{Z}\left[c_{1}, \ldots, c_{l}\right] / I^{(l, n)}$ where $I^{(l, n)}=\left(\sigma_{k+1}, \ldots, \sigma_{n-1}\right)$. In this form the variable $q$ is represented by $(-1)^{l-1} \sigma_{n}=\sigma_{\left(k+1,1^{l-1}\right)}$. In [11] other generators for the ideal $I^{(l, n)}$ are used, namely all elements $\sigma_{\lambda}$ for which $\lambda_{1}-\lambda_{l}=k+1$. It is an easy exercise to show that these elements in fact generate the same ideal, and to show that the basis $\left\{q^{d} \sigma_{\lambda} \mid \lambda_{1} \leqslant k\right\}$ of $Q H^{*} X$ is equal to the set $\left\{\sigma_{\lambda} \mid \lambda_{1}-\lambda_{l} \leqslant k\right\}$ which is the basis used in [11]. We refer to [19] for a more detailed discussion of the relations of quantum cohomology of Grassmannians with other fields.

\section{Acknowledgements}

We thank F. Sottile for triggering our search for simplifications to the theory of quantum cohomology for Grassmannians, when he explained his simplified rimhook algorithm to us. We also thank W. Fulton for numerous helpful comments to our paper, some of which greatly enhanced the clarity of our proof of the quantum Giambelli formula. Finally we thank A. Kresch for simplifying our proof of the key Lemma 1.

\section{References}

1. Bertram, A.: Quantum Schubert calculus, Adv. Math. 128 (1997), 289-305.

2. Bertram, A., Ciocan-Fontanine, I. and Fulton, W.: Quantum multiplication of Schur polynomials, J. Algebra 219 (1999), 728-746.

3. Buch, A. S., Kresch, A. and Tamvakis, H.: Gromov-Witten invariants on Grassmannians, to appear in J. Amer. Math. Soc., 2002. 
4. Buch, A. S.: A direct proof of the quantum version of Monk's formula, to appear in Proc. Amer. Math. Soc.

5. Buch, A. S.: Quantum cohomology of partial flag varieties, Preprint, 2003.

6. Ciocan-Fontanine, I.: On quantum cohomology rings of partial flag varieties, Duke Math. J. 98 (1999), 485-524.

7. Fomin, S., Gelfand, S. and Postnikov, A.: Quantum Schubert polynomials, J. Amer. Math. Soc. 10 (1997), 565-596.

8. Fulton, W.: Young Tableaux, Cambridge Univ. Press, 1997.

9. Fulton, W. and Pandharipande, R.: Notes on stable maps and quantum cohomology, In: Algebraic Geometry (Santa Cruz 1995), Amer. Math. Soc., Providence, RI, 1997, pp. 45-96.

10. Fulton, W. and Woodward, C.: On the quantum product of Schubert classes, to appear in J. Algebraic Geom.

11. Goodman, F. M. and Wenzl, H.: Littlewood-Richardson coefficients for Hecke algebras at roots of unity, Adv. Math. 82 (1990), 244-265.

12. Kac, V. G.: Infinite-Dimensional Lie Algebras, 3rd edn, Cambridge Univ. Press, 1990.

13. Kontsevich, M. and Manin, Yu.: Gromov-Witten classes, quantum cohomology, and enumerative geometry, In: Mirror Symmetry, II, Amer. Math. Soc., Providence, RI, 1997, pp. 607-653.

14. Kresch, A. and Tamvakis, H.: Quantum cohomology of the Lagrangian Grassmannian, to appear in J. Algebraic Geom.

15. Kresch, A. and Tamvakis, H.: Quantum cohomology of orthogonal Grassmannians, to appear in Compositio Math.

16. Ravi, M. S., Rosenthal, J. and Wang, X.: Degree of the generalized Plücker embedding of a Quot scheme and quantum cohomology, Math. Ann. 311 (1998), 11-26.

17. Ruan, Y. and Tian, G.: A mathematical theory of quantum cohomology, Math. Res. Lett. 1 (1994), 269-278.

18. Siebert, B. and Tian, G.: On quantum cohomology rings of Fano manifolds and a formula of Vafa and Intriligator, Asian J. Math. 1 (1997), 679-695.

19. Sottile, F.: Rational curves on Grassmannians: systems theory, reality, and transversality, Contemp. Math. 276 (2001), 9-42.

20. Walton, M. A.: Fusion rules in Wess-Zumino-Witten models, Nuclear Phys. B 340 (1990), 777-790.

21. Witten, E.: The Verlinde algebra and the cohomology of the Grassmannian, In: S. T. Yau (ed.), Geometry, Topology, and Physics, Internat. Press, Cambridge, MA, 1995, pp. $357-422$. 